\title{
REFLECTIVE $N$-PRIME RINGS WITH THE ASCENDING CHAIN CONDITION
}

BY

\author{
E. H. FELLER( ${ }^{(1)}$ AND E. W. SWOKOWSKI
}

Introduction. In an important paper [3] A. W. Goldie proved that a ring is a prime ring with certain chain conditions if and only if it has a quotient ring which is a total matrix ring $D_{n}$, where $D$ is a division ring.

For a ring $R$ let $N(R)$ denote the nil radical of $R$. We say that a ring $K$ is completely primary if $K$ has an identity and $K / N(K)$ is a division ring. In $\$ 1$ we characterize those rings $R$ which satisfy the ascending chain condition (A.C.C.) for right and left ideals and which have a quotient ring $Q(R)$ of the form $K_{n}$ where $K$ is completely primary and where $Q(R / N(R))$ $=Q(R) / N(Q(R))$. These rings are defined in $\$ 1$ as reflective $N$-prime rings.

In $\S 2$ it is proved that if $R$ is reflective $N$-prime then $R[x]$, where $x$ is a commutative indeterminate, is reflective $N$-prime.

1. Reflective $N$-prime rings. Throughout this paper, $R$ will denote a ring which satisfies the A.C.C. for right and left ideals $\left({ }^{2}\right)$. This, of course, implies that chain conditions (1) and (2) of [3] are satisfied in $R$ and $R / N(R)$. If $S$ is a subset of $R$ then $\bar{S}$ will denote the image of $S$ under the natural homomorphism from $R$ to $R / N(R)$. In particular, $\bar{R}=R / N(R)$.

Definition 1.1. A ring $R$ is termed $N$-prime if $R / N(R)$ is a prime ring.

Definition 1.2. Let $p_{1}$ and $p_{2}$ be ideals of a ring $R$. Then $R$ is called strongly $N$-prime if $p_{1} p_{2}=0, p_{1} \neq 0$ implies $p_{2} \subseteq N(R)$ and $p_{2} \neq 0$ implies $p_{1} \subseteq N(R)$.

Definition 1.3. Let $q$ be an ideal of a ring $A$. Then $A$ is said to be $q$ reflective if the following condition is satisfied: an element $a$ is regular in $A$ if and only if $a+q$ is regular in $A / q$. If $A$ is $N(\dot{A})$-reflective, then $A$ is termed reflective.

We shall say that $A$ is reflective $N$-prime if $A$ is both reflective and $N$ prime.

Statement 1.1. If $R$ is strongly $N$-prime then $R$ is $N$-prime.

Proof. Suppose $p_{1} p_{2} \subseteq N(R)$ for ideals $p_{1}$ and $p_{2}$ of $R$. Then $\left(p_{1} p_{2}\right)^{n}=0$ for some positive integer $n$. From this product let $p_{i_{1}}, p_{i_{2}}, \cdots, p_{i_{k}}$ be the smallest subcollection whose product $p_{i_{1}} p_{i_{2}} \cdots p_{i_{k}}$ is zero. Since $p_{i_{1}} \cdots p_{i_{k-1}}$ is not zero then $p_{i_{k}} \subseteq N(R)$. Thus either $p_{1}$ or $p_{2}$ is in $N(R)$ and $\bar{R}$ is prime.

This paper has been submitted to and accepted for publication by the Proceedings of the American Mathematical Society. It has been transferred to these Transactions, with the consent of the authors, for technical reasons. Presented to the Society, September 1, 1960; received by the editors June $6,1960$.

(1) Research supported by the National Science Foundation under grant, NSF-G13560.

(2) In this case, from Theorem 1 of $[5$, p. 199] $N(R)$ is a nilpotent ideal. 
Statement 1.2. If $R$ is reflective $N$-prime then $R$ is strongly $N$-prime.

Proof. Let $p_{1} p_{2}=0$ for ideals $p_{1}, p_{2}$ of $R$ and suppose $p_{1} \neq 0$ but $p_{2} \Phi N(R)$. Then $p_{2} \neq \overline{0}$ in $\bar{R}$ and by the proof of Theorem 13 of $[3$, p. 607] we know that $\bar{p}_{2}$ contains a regular element $\bar{a}$. Since $R$ is reflective, $p_{2}$ contains the regular element $a$. Thus $p_{1} p_{2} \neq 0$, a contradiction. Similarly if $p_{1} p_{2}=0$ and $p_{2} \neq 0$ then $p_{1} \subseteq N(R)$. Hence $R$ is strongly $N$-prime.

We shall now give an example of an $N$-prime ring which is not strongly $N$-prime and satisfies the A.C.C. for right and left ideals. Let $I$ denote the ring of integers. In $R=I \times I$ define $(a, b)+(c, d)=(a+c, b+d)$ and $(a, b)(c, d)$ $=(a c, a d)$. Then $N(R)=\{(0, a) \mid a \in I\}$ and $R / N(R) \cong I$. Thus $R$ is $N$-prime. However, $N(R) R=0$ and $R^{n} \neq 0$ for all positive integers $n$. Thus $R$ is not strongly $N$-prime and note that all elements of $R$ are zero divisors.

Lemma 1.1. Let $q$ be an ideal of a q-reflective ring $A$ and suppose that $A$ has at least one regular element. If $A / q$ has a right quotient ring $Q(A / q)$ then $A$ has a right quotient ring $Q(A)$. In addition, if $Q(A / q)$ is also a left quotient ring then $Q(A)$ is a left quotient ring.

Proof. Let $A^{\prime}$ denote the set of regular elements of $A$ and $\tilde{A}^{\prime}$ the set of regular elements of $\tilde{A}=A / q$. Since $A$ contains a regular element and is $q$ reflective, $A^{\prime}$ and $\tilde{A}^{\prime}$ are not empty.

Let $a$ be regular in $A$ and $b \in A, b \notin q$. Suppose $a A \cap b A^{\prime} \subseteq q$. Then $\tilde{a} \tilde{A} \cap \tilde{b} \tilde{A}^{\prime}$ $=\tilde{0}$ in $\tilde{A}$ which contradicts the fact that $Q(A)$ exists $\left({ }^{3}\right)$. Hence $a A \cap b A^{\prime} \neq 0$.

If $a$ is regular in $A$ and $b \in q$ then $a+b \notin q$ and hence there exist $u, v \in A$, $v$ regular such that $a u=(a+b) v$. Thus $a(u-v)=b v$ and $a A \cap b A^{\prime} \neq 0$. It follows from $[3$, p. 118] that $A$ has a right quotient ring $Q(A)$.

If $Q(A / q)$ is also a left quotient ring then from a discussion similar to that above, for $a, b \in A, a$ regular, there exist $u, v \in A, v$ regular such that $u a=v b$. Hence if $x=b a^{-1}$ in $Q(A)$ then $x=v^{-1} u$. Thus $Q(A)$ is also a left quotient $\operatorname{ring}\left({ }^{4}\right)$.

If $S$ is a ring extension of $A$ and if $h$ is an ideal of $A$ then $h^{*}$ will denote the extension of $h$ to $S$. If $k$ is an ideal in $S$ then $k_{*}$ denotes the contraction of $k$ in $A$.

Lemma 1.2. Let $A$ be q-reflective where $q$ is an ideal of $A$ and suppose that $A$ has at least one regular element. If $A / q$ has a right quotient ring $Q(A / q)$ then $q^{*}=\left\{a b^{-1} \mid a \in q, b\right.$ regular in $\left.A\right\}$ and $Q(A / q) \cong Q(A) / q^{*}$. Moreover $\left(q^{*}\right)_{*}=q$.

Proof. By Lemma 1.1, $A$ has a right quotient ring $Q(A)$. Let $T=\left\{a b^{-1} \mid a \in q\right.$,

${ }^{(3)}$ Here, and in the proof of Lemma 1.2, $\tilde{a}$ denotes the image of $a$ under the natural homomorphism from $A$ to $\tilde{A}$.

(4) As pointed out by Goldie [3, p. 604] one need not assume that $A$ has an identity element. Note also that if $a^{\prime \prime}$ denotes the elements of $Q(A)$ of the form $(a c) c^{-1}$ then the mapping $a \rightarrow a^{\prime \prime}$ is an isomorphism of $A$ onto $A^{\prime \prime}$, a subring of $Q(A)$. We shall identify $A$ with $A^{\prime \prime}$ and consider $A$ as a subring of $Q(A)$. 
$b$ regular in $A\}$. For any $a b^{-1}, c d^{-1} \in T$ there exist regular elements $b_{1}, d_{1} \in A$ such that $m=d b_{1}=b d_{1}$. Then $a b^{-1}-c d^{-1}=\left(a d_{1}-c b_{1}\right) m^{-1}$ is in $T$ since $a, c \in q$. Suppose $e f^{-1}$ is an element of $Q(A)$. If $e_{1}, b_{1} \in A, b_{1}$ regular and $e b_{1}=b e_{1}$ we have $\left(a b^{-1}\right)\left(e f^{-1}\right)=\left(a e_{1}\right)\left(f b_{1}\right)^{-1} \in T$. Similarly if $a_{1}, f_{1} \in A, f_{1}$ regular and $a f_{1}$ $=f a_{1}$, then $\left(e f^{-1}\right)\left(a b^{-1}\right)=\left(e a_{1}\right)\left(b f_{1}\right)^{-1}$ which is in $T$ since $a_{1} \in q$. Hence $T$ is an ideal in $Q(A)$ and is clearly the smallest ideal in $Q(A)$ which contains $q$. Hence $T=q^{*}$.

It is readily verified that the mapping $a b^{-1} \rightarrow \tilde{a} \tilde{b}^{-1}$ is a homomorphism of $Q(A)$ onto $Q(A / q)$ with kernel $q^{*}$. Consequently $Q(A / q) \cong Q(A) / q^{*}$.

To prove the last part of the lemma it is sufficient to show that $\left(q^{*}\right)_{*} \subseteq q$. Let $b \in\left(q^{*}\right)_{*}=q^{*} \cap A$. Then $b=a c^{-1}$ where $a \in q$ and $c$ is regular in $A$. It follows that $\tilde{b} \tilde{c}=\tilde{a}=\tilde{0}$ in $\tilde{A}$ and since $\tilde{c}$ is regular we conclude that $b \in q$.

THEOREM 1.1. If $R$ is reflective $N$-prime then $R$ has a right quotient ring $Q(R)$ which is also a left quotient ring.

Proof. We know by the corollary to Theorem 10 of [3] that $\bar{R}$ contains a regular element and thus $R$ contains a regular element. By Theorem 11 of [3], $R / N(R)$ has a right quotient ring which is also a left quotient ring. Hence the conditions of Lemma 1.1 are satisfied.

Lemma 1.3. Let $R$ be a reflective $N$-prime ring. If $k$ and $h$ are right (or left) ideals of $Q(R)$ and $k \subset h$ then $k_{*} \subset h_{*}\left({ }^{(}\right)$.

Proof. Suppose $a b^{-1} \in h$ and $a b^{-1} \notin k$ where $a, b \in R, b$ regular. Then $\left(a b^{-1}\right) b=a \in h \cap R=h_{*}$. If $a \in k_{*}$ then $a b^{-1} \in k$, a contradiction. Hence $k_{*} \subset h_{*}$. A similar argument holds if $k$ and $h$ are left ideals.

From this lemma we have at once:

Statement 1.3. If $R$ is reflective $N$-prime then $Q(R)$ satisfies the A.C.C. for right and left ideals.

Statement 1.4. If $R$ is reflective $N$-prime then $N(Q(R))=N(R)^{*}$ and $\bar{R}$ has a right and left quotient ring $Q(\bar{R})$. Moreover $Q(\bar{R}) \cong[Q(R)]-$.

Proof. Applying Lemma 1.2 we have $N(R)^{*}=\left\{a b^{-1} \mid a \in N(R), b\right.$ regular in $R\}$. If $k$ is an ideal of $Q(R)$ and $k \supset N(R)^{*}$ then, by Lemmas 1.3 and 1.2, $k_{*} \supset\left(N(R)^{*}\right)_{*}=N(R)$. From the proof of Theorem 13 of [3] we know that $k_{*}$ contains a regular element and consequently $k$ contains a regular element. Hence $k$ is not nilpotent. Thus $N(R)^{*}$ is a maximal nilpotent ideal and $N(R)^{*}$ $=N(Q(R))$.

By Theorem 13 of [3], $\bar{R}$ has a right and left quotient ring $Q(\bar{R})$ which is a total matrix ring over a division ring. Using Lemma 1.2 we may write $Q(\bar{R}) \cong Q(R) / N(Q(R))=[Q(R)]^{-\left({ }^{(}\right)}$.

(5) The symbol $C$ denotes proper inclusion.

(6) Previously we have considered $R$ as a subring of $Q(R)$. Now since $N(R)=N(Q(R)) \cap R$ we shall consider $\bar{R}$ as a subring of $[Q(R)]^{-}$and say that $[Q(R)]^{-}=Q(\bar{R})$. In the future when we write $[Q(R)]^{-}=Q(\bar{R})$ we tacitly assume the above situation. 
A ring $K$ is said to be completely primary if $K$ has an identity and $K / N(K)$ is a division ring. Since $K / N(K)$ is simple, the Jacobson radical of $K$ equals $N(K)$. We can now apply Proposition 3 of $[5$, p. 54] and Theorem 1 of $[5$, p. 56] to prove

THEOREM 1.2. If $R$ is reflective $N$-prime then $Q(R)$ is isomorphic to a total matrix ring $K_{n}$ where $K$ is a completely primary ring.

Note that since $Q(R) \cong K_{n}$ satisfies the A.C.C. for right and left ideals then so does $K$. Conversely if $K$ satisfies the A.C.C. for right and left ideals then certainly this is true in $K_{n}$.

Statement 1.5. If $K$ is a completely primary ring which satisfies the A.C.C. for right and left ideals, then $K_{n}$ is reflective $N$-prime.

Proof. Since the only ideals of $K_{n}$ are of the form $H_{n}$ where $H$ is an ideal of $K$ then $N\left(K_{n}\right)=(N(K))_{n}$. Since $\bar{K}_{n}$ is simple certainly $K_{n}$ is $N$-prime( $\left.{ }^{7}\right)$.

If $\bar{A}$ is a regular element in $\bar{K}_{n}$, then by Theorem 2 of $[4$, p. 20] we know that $\bar{A}$ is a unit of $\bar{K}_{n}$. From 2a of [1] we have that $A$ is a unit of $K_{n}$ and is therefore regular.

Suppose $A=\left(a_{i j}\right)$ is regular in $K_{n}$ but $\bar{A}$ is not regular in $\bar{K}_{n}$. Then in $\bar{K}_{n}$ there exists a unit $\bar{U}$ such that $\bar{U} \bar{A}$ is a matrix in which every element in row $n$ is $\overline{0}$. As above $U$ is a unit of $K_{n}$ and $U A=\left(c_{i j}\right)$ is a matrix with $c_{n j} \in N$ $=N(K), j=1,2, \cdots, n$. Let $B=\left(b_{i j}\right)$ where $b_{i j}=0, j \neq n$ and $0 \neq b_{i n} \in N_{n}^{t-1}$ where $N_{n}^{t-1} \neq 0$ and $N_{n}^{t}=0$. Then $B U A=0$ which implies that $B U=0$, which in turn implies that $B=0$, a contradiction.

From this proof we have the

Corollary. If $K$ is a completely primary ring which satisfies the A.C.C. for right and left ideals then $A \in K_{n}$ is a unit if and only if $A$ is not a right (left) zero divisor.

THEOREM 1.3. If $R$ has a right and left quotient ring $Q(R)$ of the form $K_{n}$, where $K$ is completely primary and if $Q(\bar{R}) \cong \bar{K}_{n}$, then $R$ is reflective $N$-prime.

Proof. By 2a of [1] an element is a unit of $K_{n}$ if and only if it is a unit of $\bar{K}_{n}$. Thus an element is regular in $R$ if and only if it is regular in $\bar{R}$.

Since $Q(\bar{R}) \cong \bar{K}_{n} \cong(\bar{K})_{n}$ we have by Theorem 13 of $[3]$ that $\bar{R}$ is prime. Hence $R$ is $N$-prime.

We summarize our results as follows:

TheOREM 1.4. If $R$ satisfies the A.C.C. for right and left ideals then the following statements are equivalent.

1. $R$ is reflective $N$-prime.

2. $R$ has a right and left quotient ring $Q(R)$ of the form $K_{n}$, where $K$ is a completely primary ring which satisfies the A.C.C. for right and left ideals. In addition $Q(\bar{R}) \cong \bar{K}_{n}$.

${ }^{(7)}$ The symbol $\bar{K}_{n}$ denotes the ring $\left[\left(K_{n}\right)\right]^{-}$. Note that $\bar{K}_{n} \cong(\bar{K})_{n}$. 
2. The polynomial ring $R[x]$. In this section, in addition to the A.C.C. for right and left ideals, we shall assume that $R$ has an identity element 1 . Let $x$ be a commutative indeterminate. We shall show that if $R$ is prime then $R[x]$ is prime and if $R$ is reflective $N$-prime then $R[x]$ is also reflective $N$ prime. These results provide a means by which one can properly extend a prime ring or a reflective $N$-prime ring to a ring of the same type.

In the course of establishing these results we shall prove several statements about matrix rings which are of some interest in themselves.

THEOREM 2.1. If $R$ is a prime ring, then $R[x]$ is a prime ring.

Proof. If $H \neq 0$ is an ideal of $R[x]$, let $q(H)$ denote the set of all coefficients of the least power of $x$ that appears in $H$ with nonzero coefficients $\left({ }^{8}\right)$. Then $q(H)$ is a nonzero ideal in $R$. If $J \neq 0$ is an ideal of $R[x]$ and $H J=0$ then $q(H) q(J)=0$ in $R$, a contradiction.

Theorem 2.2. If $R$ is $N$-prime then $N[x]=N(R[x])$ and $R[x]$ is $N$-prime.

Proof. Certainly $N[x] \subseteq N(R[x])$ and we have by Theorem 2.1 that $R[x] / N[x]=(R / N)[x]$ is a prime ring. Hence $N[x]$ is a maximal nilpotent ideal which implies that $N[x]=N(R[x])$.

In Chapter 3 of [4], for the elements of the matrix ring $P_{n}, P$ a principal ideal domain, there is defined an equivalence relation $(\sim)$. Here $A \sim B$ in $P_{n}$ if and only if there exist units $U$ and $V$ in $P_{n}$ such that $A=U B V$. If $A \sim B$ then $A$ and $B$ are termed associates. From the discussion on page 42 of this chapter we have that every matrix $A$ has an associate $D=\left(d_{i j}\right)$ where $d_{i i}$ is a total divisor of $d_{j j}$ for $j>i$ and $d_{i j}=0, i \neq j$. Certainly if $d_{j j}=0$ then $d_{k k}=0$ for $k>j$. We shall call this matrix $D$ the canonical form of $A$. From page 31 of [4] if $P$ is a principal ideal domain then the right quotient ring $Q(P)$ exists. Surely $Q(P)$ is also a left quotient ring and a division ring.

Statement 2.1. Let $P$ be a principal ideal domain and $Q(P)$ its quotient ring. Let $A$ be an element of $P_{n}$ with canonical form $D=\left(d_{i j}\right)$. Then $A$ is a right divisor of zero if and only if $d_{n n}=0$. In this case $A$ is also a left divisor of zero.

Proof. If $d_{n n} \neq 0$ in the canonical form $D$, then $d_{i i} \neq 0$ for all $i$. Hence in $Q(P)_{n}$ the matrix $D$ is a unit. Thus if $U A V=D$ then $A=U^{-1} D V^{-1}$ is a unit of $Q(P)_{n}$ and not a divisor of zero in $P_{n}$.

If now $d_{n n}=0$ in $D$, then one can easily find a nonzero matrix $B$ such that $D B=0$. Then for $U A V=D$, we have $A V B=U^{-1} D B=0$ where $V B \neq 0$ since $V$ is a unit. Thus $A$ is a right divisor of zero. Similarly $A$ is a left divisor of zero.

If $R / N(R)$ is a principal ideal domain then in $R_{n}$ one can define an equivalence relation $(\sim)$ as before. We write $A \sim B$ in $R_{n}$ if and only if there exist units $U$ and $V$ in $R_{n}$ such that $A=U B V$. If $A \sim B$ then $A$ and $B$ are called

$\left.{ }^{8}\right)$ For the purposes of this proof consider $x^{0}=1$. 
associates. From 2a of [1] we know that $U$ is a unit of $R_{n}$ if and only if $\bar{U}$ is a unit of $\bar{R}_{n}$. From the preceding discussion we know that every matrix $A$ has an associate $D=\left(d_{i j}\right)$ where $\left[d_{i i}\right]-$ is a total divisor of $\left[d_{j j}\right]-$ in $\bar{R}$ for $j>i$ and $d_{i j} \in N(R)$ for $i \neq j$. Certainly if $d_{j j} \in N(R)$ then $d_{k k} \in N(R)$ for $k>j$. In this case we call this matrix $D$ the canonical form of $A$.

Statement 2.2. Suppose $R / N(R)$ is a principal ideal domain where $R$ has a right and left quotient ring $Q(R)$ and $[Q(R)]^{-}=Q(\bar{R})$. Let $A$ be a matrix in $R_{n}$ with canonical form $D=\left(d_{i j}\right)$. Then $A$ is a right divisor of zero if and only if $d_{n n} \in N(R)$. In this case $A$ is a left divisor of zero. In addition $R_{n}$ is reflective.

Proof. If $d_{n n} \notin N$ then we have, by the proof of Statement 2.1, that $\bar{A}$ is a unit of $[Q(R)]_{n}^{-}$. From $2 \mathrm{a}$ of $[1]$ it follows that $A$ is a unit in $Q(R)_{n}$ and hence is not a divisor of zero in $R_{n}$.

If $d_{n n} \in N$, let $B=\left(b_{i j}\right)$ be a matrix with $b_{i j}=0$ for $i \neq n$ and $0 \neq b_{n j} \in N^{\imath-1}$ for $j=1,2, \cdots, n$, where $N^{t}=0$ and $N^{t-1} \neq 0$. Then $D B=0$ and, as in the proof of Statement 2.1, $A$ is a right and left divisor of zero.

The fact that $R_{n}$ is reflective follows from this discussion.

Corollary. If $K$ is a completely primary ring which satisfies the A.C.C. for right ideals then $(K[x])_{n}$ is reflective.

Proof. By [2] we know that $Q(K[x])$ exists and $[Q(K[x])]^{-}=Q[(K[x])]^{-}$.

THEOREM 2.3. If $R / N(R)$ is a principal ideal domain where $R$ has a right and left quotient ring $Q(R)$ and $Q(\bar{R})=[Q(R)]^{-}$then $(Q(R))_{n}$ is a right and left quotient ring of $R_{n}$.

Proof. From Statement 2.2 and its proof we know that if $A$ is regular in $R_{n}$ then $A$ has an inverse in $(Q(R))_{n}$. To prove that $R$ has a right quotient ring we need only show that every element of $(Q(R))_{n}$ is of the form $A B^{-1}$ where $A, B \in R_{n}$. We shall prove this for the case $n=2$. The method of proof can easily be extended to the general case. Thus consider

$$
A=\left(\begin{array}{ll}
a b^{-1} & c d^{-1} \\
e f^{-1} & g h^{-1}
\end{array}\right) \in(Q(R))_{2} .
$$

Let

$$
B=\left(\begin{array}{ll}
b & 0 \\
0 & 1
\end{array}\right)
$$

Then $B$ is regular and

$$
A B=\left(\begin{array}{cc}
a & c d^{-1} \\
e f^{-1} b & g h^{-1}
\end{array}\right) .
$$

Since $Q(R)$ is a right quotient ring we may write $e f^{-1} b=r s^{-1}$. Now multiply $A B$ by 


$$
C=\left(\begin{array}{ll}
s & 0 \\
0 & 1
\end{array}\right)
$$

to obtain

$$
A B C=\left(\begin{array}{cc}
\text { as } & c d^{-1} \\
r & g h^{-1}
\end{array}\right) .
$$

Similarly, multiplying $A B C$ on the right by

$$
D=\left(\begin{array}{ll}
1 & 0 \\
0 & d
\end{array}\right)
$$

then substituting $t v^{-1}$ for $g h^{-1} d$ and multiplying the resulting matrix on the right by

$$
E=\left(\begin{array}{ll}
1 & 0 \\
0 & v
\end{array}\right)
$$

we obtain a set of matrices whose product $F$ is regular such that $A F=G \in R_{2}$. Thus $A=G F^{-1}$.

It is easy to see that $(Q(R))_{n}$ is also a left quotient ring.

THEOREM 2.4. Let $A$ be a ring with right and left quotient ring $Q(A)$. Then $f(x)$ is regular in $A[x]$ if and only if $f(x)$ is regular in $Q(A)[x]$.

Proof. The "if" part is immediate. To prove the "only if" part we shall use only polynomials of degree one. The method obviously extends to polynomials of any degree.

Suppose $(a x+b)$ is regular in $A[x]$ but not in $Q(A)[x]$. Suppose $(a x+b)\left(c d^{-1} x+e f^{-1}\right)=0$ where we may assume that $c d^{-1} \neq 0$. Thus

$$
\begin{aligned}
(a x+b)\left(c x+e f^{-1} d\right) & =0 \\
(a x+b)\left(c x+g h^{-1}\right) & =0 \\
(a x+b)(c h x+g) & =0
\end{aligned}
$$

where $c h \neq 0$ since $h$ is regular, a contradiction. Similarly $\left(c d^{-1} x+e f^{-1}\right)(a x+b)$ $\neq 0$ for every $c d^{-1} \neq 0$.

THEOREM 2.5. If $R$ is a reflective $N$-prime ring then $R[x]$ is a reflective $N$ prime ring.

Proof. By Theorem 2.2, it is sufficient to show that when $R$ is reflective then $R[x]$ is reflective. We know that $R$ has a right and left quotient ring $Q(R)$ and moreover that $Q(R)$ is of the form $K_{n}$ where $K$ is a completely primary ring which satisfies the A.C.C. for right and left ideals. Thus we may say $R[x] \subseteq K_{n}[x]$. From Statement 1.4 we have that $Q(\bar{R})=\bar{K}_{n}$ and conse- 
quently we write $\bar{R}[x] \subseteq \bar{K}_{n}[x]$. Then the corollary to Statement 2.2 tells us that $K_{n}[x]$ is reflective. The result now follows by applying Theorem 2.4 .

The results given in this paper provide a method for properly extending the prime rings $D_{n}$ where $D$ is a division ring or the reflective $N$-prime rings $K_{n}$ where $K$ is a completely primary ring with A.C.C. for right and left ideals to rings of the same type. Thus, from Theorem 2.3 and the preceding discussion we have

$$
D_{n} \subset D_{n}[x]=(D[x])_{n} \subset Q\left((D[x])_{n}\right)=(Q(D[x]))_{n}
$$

and

$$
K_{n} \subset K_{n}[x]=(K[x])_{n} \subset Q\left((K[x])_{n}\right)=(Q(K[x]))_{n} .
$$

The existence of $Q(D[x])$ is proved in [4, Chapter 3] and the existence of $Q(K[x])$ is given in [2].

\section{BIBLIOGRAPHY}

1. E. H. Feller, Properties of primary noncommutative rings, Trans. Amer. Math. Soc. vol. 89 (1958) pp. 79-91.

2. E. H. Feller and E. W. Swokowski, On ring extensions of completely primary noncommutative rings, unpublished.

3. A. W. Goldie, The structure of prime rings under ascending chain conditions, Proc. London Math. Soc. vol. 8 (1958) pp. 589-608.

4. N. Jacobson, The theory of rings, Mathematical Surveys, no. 2, American Mathematical Society, 1943.

5. - Structure of rings, Amer. Math. Soc. Colloquium Publications, vol. 37, 1956.

UNIVERSITY OF WISCONSIN,

MILWAUKEE, WisCONSIN

MARQUETTE UNIVERSITY,

MiLWAUKEE, WisCoNSIN 